\title{
Phantom Preparation Using a Dilution Technique
}

\author{
Martin A. Lodge \\ Johns Hopkins University, Baltimore, Maryland
}

$\mathbf{P}$

hysical phantoms with compartments containing different concentrations of radioactivity are widely used in PET methodology research, system performance assessment, and routine quality assurance. Accreditation bodies such as the American College of Radiology require the use of a phantom involving an aqueous solution of ${ }^{18} \mathrm{~F}$ in various inserts and a lower radioactivity concentration in a background region. These phantoms have to be prepared manually, usually by technologists or physicists with specific experience in this kind of experimental work. Even in the hands of experienced practitioners, phantom preparation is prone to experimental error and subject to variability because it involves many manual steps. When unexpected phantom results occur, they are commonly attributed to fluctuations in scanner performance but in many cases may simply be due to errors in phantom filling. Reliable phantom preparation is clearly important, and in this article, readers are reminded of a dilution technique that can greatly simplify the process.

Current guidance on phantom filling typically involves preparation of 2 carefully calibrated radioactive samples (1). One is transferred to the phantom background compartment, and the other is used to prepare a higher-concentration solution that is subsequently used to fill the phantom inserts. With knowledge of the volumes of the relevant compartments, target activities can be selected that provide the required insert-to-background concentration ratio. Although conceptually straightforward, in practice this process is complicated by the difficulty of preparing 2 rapidly decaying samples with precise activities at the same time. To allow for this difficulty, each radioactivity measurement is permitted to deviate from the target value within a certain range, but this flexibility can introduce large errors in the resulting insertto-background ratio and unwanted variability between repeated experiments. Skilled operators are adept at managing these complexities, but more accurate and repeatable phantom preparation can be achieved with a simpler filling protocol.

An alternative to the procedure described above involves an initial step in which the phantom background compartment is partially filled with water. To achieve an N-to-1 insert-tobackground radioactivity concentration ratio, the phantom would initially be filled with $1 / \mathrm{N}$ of the total background volume, for example, 4/10 full in the case of a 2.5-to-1 ratio. A single calibrated radioactive sample is then added to the phantom background, and after thorough mixing, a small volume of this

radioactive solution is withdrawn and used to fill the phantom inserts. Finally, the phantom background compartment is completely filled with additional nonradioactive water, diluting the background radioactivity concentration and thereby achieving the desired insert-to-background ratio. Allowance can be made for the small volume of liquid used to fill the inserts by adding an equivalent volume to the initial fill. Also, the small amount of radioactivity withdrawn to fill the inserts can be estimated from the relevant volumes and subtracted from the dose calibrator measurement to determine the final activity in the background region.

This dilution technique is readily applicable to many different phantoms commonly used in PET, including the National Electrical Manufacturers Association NU2 image-quality phantom and the American College of Radiology PET phantom (Data Spectrum). As an example, the latter phantom was prepared with a 2.5-to-1 insert-to-background ratio on 5 separate occasions. One-time volume measurements of the background compartment $(5,680 \mathrm{~mL})$ and 4 fillable inserts $(33 \mathrm{~mL}$ total) were determined by weighing with an accurate balance. Phantom filling was tested by withdrawing $300-\mu \mathrm{L}$ aliquots from the insert and background compartments and measuring with a $\mathrm{NaI}(\mathrm{Tl}) \gamma$-counter. The mean ( \pm SD of repeated measurements) insert-to-background radioactivity concentration ratio derived from the $\gamma$-counter was $2.48 \pm 0.01$. Relative to the target ratio of 2.5 , this corresponds to $99.3 \% \pm 0.5 \%$, suggesting highly accurate and highly repeatable phantom preparation. The method is easy to implement, can be performed rapidly, and is highly robust because the insert-tobackground ratio is not dependent on any dose calibrator measurements. Although dilution methods of this sort have no doubt been in use for some time, more widespread awareness may be helpful and the technique could be considered further as phantom guidelines are updated.

\section{DISCLOSURE}

No potential conflict of interest relevant to this article was reported.

\section{REFERENCE}

1. Phantom testing: PET. ACR accreditation website. https://accreditationsupport. acr.org/support/solutions/articles/11000062800-phantom-testing-pet. Published December 12, 2019. Accessed December 10, 2020.

Received Sep. 18, 2020; revision accepted Sep. 22, 2020.

For correspondence and reprints contact: Martin A. Lodge, Radiology/ Nuclear Medicine, Johns Hopkins Hospital, 600 N. Wolfe St., Baltimore, MD 21287.

E-mail: mlodge1@jhmi.edu

Published online Dec. 11, 2020.

COPYRIGHT ( 2021 by the Society of Nuclear Medicine and Molecular Imaging.

DOI: 10.2967/jnumed.120.257097 